\title{
Hadron production from quark coalescence and jet fragmentation
}

\author{
V. Greco and C. M. Ko \\ Cyclotron Institute and Physics Department, Texas A63M University, College Station, Texas 77843-3366
}

I. Vitev

Theory Division and Physics Division, Los Alamos National Laboratory, Mail Stop H846, Los Alamos, New Mexico 87545

(Received 16 December 2004; published 26 April 2005)

\begin{abstract}
Transverse momentum spectra of pions, protons, and antiprotons in $\mathrm{Au}+\mathrm{Au}$ collisions at intermediate RHIC energy $\sqrt{s_{N N}}=62 \mathrm{GeV}$ are studied in a model that includes both quark coalescence from the dense partonic matter and fragmentation of the quenched perturbative minijet partons. The resulting baryon to meson ratio at intermediate transverse momenta is predicted to be larger than that seen in experiments at higher center of mass energies.
\end{abstract}

DOI: 10.1103/PhysRevC.71.041901

PACS number(s): 25.75.Dw, 25.75.Nq, 12.38.Bx

Recently, there has been renewed interest in using the quark coalescence or recombination model to study hadron production in ultrarelativistic heavy ion reactions [1-9]. These studies were largely triggered by two surprising observations in measured hadron spectra from $\mathrm{Au}+\mathrm{Au}$ collision at $\sqrt{s_{N N}}=200 \mathrm{GeV}$ at the BNL Relativistic Heavy Ion Collider (RHIC) [1,10-12]. The first was the nearly similar yield of protons and pions at intermediate transverse momentum $\left(2 \mathrm{GeV}<p_{T}<5 \mathrm{GeV}\right)$, which is at variance with the expectation from the fragmentation of minijets produced from initial hard processes. The second was the so-called "quark number scaling" of hadron elliptic flow $v_{2 h}\left(p_{T}\right)$, i.e., the transverse momentum dependence becomes universal if both $v_{2 h}$ and transverse momentum $p_{T}$ are divided by the number of constituent quarks in the hadron. Both phenomena have a simple explanation if hadronization of the thermally equilibrated and collectively flowing partonic matter formed in these collisions proceeds through the coalescence of quarks of constituent masses [3-6].

The description of hadronization of the quark-gluon plasma formed in heavy ion collision at relativistic energies by quark coalescence was first introduced in models such as ALCOR [13] and MICOR [14]. Emphases of these earlier studies were on particle yields and their ratios. Recent studies have been more concerned with observables that are related to collective dynamics and production of hadrons with relatively large transverse momenta. Furthermore, effects of minijet partons from initial hard processes have also been included through their independent fragmentation as well as coalescence with soft partons in the quark-gluon plasma $[3,4]$. The latter provides a new mechanism for the hadronization of minijet partons produced in relativistic heavy ion collisions. The interplay between quark coalescence and minijet fragmentation has been found to be essential for understanding measured inclusive hadron spectra at moderate and high transverse momenta. In particular, the observed jetlike features of these hadrons [15] seem to also support the scenario that they are produced from the coalescence of minijets with the thermal partons in the produced quark-gluon plasma. If confirmed by more exclusive data from experiments and by further theoretical studies, hadronization via quark coalescence then represents a new probe of the quark-gluon plasma formed in relativistic heavy ion collisions, and its hadronization mechanism, as well as that of the minijet partons produced in these collisions. It is thus of great interest to study the onset of the coalescence mechanism as the collision energy is varied. Also, comparison of results from the coalescence model for heavy ion collisions at different energies with upcoming experimental data will allow for a better understanding of its predictive power. In the present paper, such a study is carried out by using the same coalescence model as applied previously to higher energy collisions to make predictions for the proton, antiproton, and pion spectra at a lower collision energy of $\sqrt{s_{N N}}=62 \mathrm{GeV}$.

The coalescence model describes the dynamical process of converting quarks and antiquarks into hadron bound states in the presence of partonic matter. In general, the process of coalescence involves the emission of a third particle or, more generally, offshell effects to guarantee energy conservation and color neutrality. However, under the assumption of a fast process in which the binding interaction is turned on suddenly, the probability of forming the bound state is simply given by the overlap of the quark and antiquark distribution functions with the Wigner function of the formed hadron. In this case, the transverse momentum spectrum of a hadron consisting of $n$ (anti)quarks can be written as

$$
\begin{aligned}
\frac{d N_{H}}{d^{2} P_{T}}= & g_{H} \int \prod_{i=1}^{n} \frac{d^{3} \mathbf{p}_{i}}{(2 \pi)^{3} E_{i}} p_{i} \cdot d \sigma_{i} f_{q}\left(x_{i}, p_{i}\right) \\
& \times f_{H}\left(x_{1} \ldots x_{n} ; p_{1} \ldots p_{n}\right) \delta^{(2)}\left(P_{T}-\sum_{i=1}^{n} p_{T, i}\right)
\end{aligned}
$$

In the above, $d \sigma$ denotes an element of a spacelike hypersurface, and $g_{H}$ is the statistical factor for forming a colorless hadron from colored quarks and antiquarks of spin $1 / 2$. For hadrons considered here, i.e., $\pi, \rho, K^{*}, p$, and $\Delta$, the statistical factors are, respectively, $g_{\pi}=g_{\omega}=1 / 36, g_{\rho}=$ $g_{K^{*}}=1 / 12, g_{p}=1 / 108$, and $g_{\Delta}=1 / 54$. The $n$-quark phase space distribution function has been approximated in Eq. (1) by the product of the single quark distribution function $f_{q}(x, p)$ that is normalized to the quark number in the partonic matter. 
The Wigner function $f_{H}\left(x_{i} ; p_{i}\right)$ in Eq. (1) describes the spatial and momentum distributions of quarks in a hadron. For mesons, we have taken the Wigner functions to be a sphere of radius $\Delta_{x}$ in coordinate space and $\Delta_{p}=\Delta_{x}^{-1}$ in momentum space, i.e.,

$$
\begin{aligned}
f_{M}\left(x_{1}, x_{2} ; p_{1}, p_{2}\right)= & \frac{9 \pi}{2} \Theta\left[\Delta_{x}^{2}-\left(x_{1}-x_{2}\right)^{2}\right] \\
& \times \Theta\left[\Delta_{p}^{2}-\left(p_{1}-p_{2}\right)^{2}+\left(m_{1}-m_{2}\right)^{2}\right] .
\end{aligned}
$$

This represents the simplest ansatz for the Wigner function, i.e., it gives a probability of 1 if the relative covariant distances between two particles in space and momentum are smaller than the radius parameters. The Wigner functions for baryons can be similarly expressed in terms of appropriate relative coordinates (see Ref. [4] for detail). Although hadron wave functions are generally not known in detail, the particular shape of the wave function used in our model does not significantly affect the result on hadron transverse momentum spectra, unless very extreme choices are taken. As in our previous work [3], we take $\Delta_{p}=0.24 \mathrm{GeV}$ for mesons and $\Delta_{p}=0.36 \mathrm{GeV}$ for baryons in the present study.

One of the main ingredients in our model is the parton distribution functions at hadronization. They can in principle be determined from a dynamic approach such as the transport model [16-20]. To focus on the hadronization process itself, we assume instead that at hadronization the partonic matter consists of both a thermally and chemically equilibrated quarkgluon plasma and quenched minijet partons from initial hard processes. Specifically, the single quark distribution function is taken to consist of both a thermal and a minijet component, separated by the transverse momentum $p_{0} \sim 2 \mathrm{GeV}$. In this section, we discuss how the thermal component is determined from measured total transverse energy as in our previous work at $\sqrt{s_{N N}}=200 \mathrm{GeV}$ [4], whereas the minijet one is discussed in the next section together with the minijet fragmentation process.

Under the assumption that the quark-gluon plasma is thermally equilibrated and collectively flowing, the determination of its parton distributions requires knowledge of the radial flow and its total transverse energy. A blast wave analysis of the experimental data shows that the collective radial flow differs very little between the highest CERN Super Proton Synchrotron (SPS) and RHIC energies. We can therefore assume the same radial flow for $\mathrm{Au}+\mathrm{Au}$ collisions at $\sqrt{s_{N N}}=62 \mathrm{GeV}$ as at higher RHIC energies, i.e., $\beta_{\max }=0.5$, or a slightly smaller one of about 0.45 , and the usual linear radial profile $\beta=\beta_{\max } r / R$, where $R$ is the transverse size of the fireball. We have checked that this uncertainty does not have a significant effect on the transverse momentum dependence of the $p / \pi$ ratio, which is the main observable we want to study in this paper.

As to the total transverse energy, we are not aware at the moment of any measurement for $\mathrm{Au}+\mathrm{Au}$ collisions at $\sqrt{s_{N N}}=62 \mathrm{GeV}$. It has been noticed, however that the ratio of the transverse energy to the total number of charged particles in relativistic heavy ion collisions is nearly constant in the energy range $20 \mathrm{GeV}<\sqrt{s_{N N}}<200 \mathrm{GeV}$, and available experimental data [21] can be parametrized by

$$
\frac{d E_{T}}{d N_{\mathrm{ch}}}=0.88+1.44 \times 10^{-3}\left(\sqrt{s_{N N}}-130\right) \mathrm{GeV}
$$

Also, the charged particle multiplicity normalized to the number of participants has a smooth logarithmic behavior that can be parametrized as

$$
\frac{d N_{\mathrm{ch}}}{d \eta\left(N_{\mathrm{part}} / 2\right)}=0.37+0.62 \ln \sqrt{s_{N N}} .
$$

Using the Glauber model to estimate the number of participants, we have $N_{\text {part }}=330$ for the $0-10 \%$ centrality bin. From Eq. (4), the charged particle multiplicity is then $d N_{\mathrm{ch}} / d \eta=480$, corresponding to a multiplicity per unit of rapidity of $d N_{\mathrm{ch}} / d y=1.2 d N_{\mathrm{ch}} / d \eta=580$, with $d \eta / d y=$ 1.2 from the relation between rapidity and pseudorapidity. Equation (3) thus leads to a transverse energy $d E_{T} / d y \simeq$ $460 \mathrm{GeV}$ for central $\mathrm{Au}+\mathrm{Au}$ collisions at $\sqrt{s_{N N}}=$ $62 \mathrm{GeV}$.

What remains to be determined is the relative abundance of quarks and antiquarks, which is essentially given by the baryon chemical potential. This can in principle also be extracted from experimental data on the $\bar{p} / p$ ratio via $\mu_{q}=T / 6 \ln \left(N_{p} / N_{\bar{p}}\right)$. For collisions at $\sqrt{s_{N N}}=200 \mathrm{GeV}$, we already had an experimental value of $\bar{p} / p \simeq 0.7$, which corresponds to a quark chemical potential $\mu_{q}=11 \mathrm{MeV}$ [4]. There is no measurement of the $\bar{p} / p$ ratio yet at $\sqrt{s_{N N}}=62 \mathrm{GeV}$, but it can be estimated from a parametrization of the chemical potential in the energy range of $10 \mathrm{GeV}<\sqrt{s_{N N}}<200 \mathrm{GeV}$, based on available data from BNL Alternating Gradient Synchrotron (AGS) to RHIC. The resulting quark chemical potential is $\mu_{q}=23 \mathrm{MeV}$, leading to an antiproton to proton ratio $\bar{p} / p=0.44$. Because of strangeness conservation, numbers of strange and antistrange quarks are equal, i.e., there is zero strangeness chemical potential. The relative abundance of strange to light quark is then simply determined by their constituent quark masses that are taken to be $m_{u, d}=300 \mathrm{MeV}$ and $m_{s}=475 \mathrm{MeV}$. Assuming a critical temperature of $T_{c}=170 \mathrm{MeV}$, the partonic matter at hadronization is found to have $N_{u+d}=285, N_{\bar{u}+\bar{d}}=218$, and $N_{s}=N_{\bar{s}}=79$ quarks and antiquarks distributed in a volume $V=555 \mathrm{fm}^{3}$ in order to have the estimated transverse energy of $460 \mathrm{GeV}$. This leads to a critical energy density of about $0.8 \mathrm{GeV} / \mathrm{fm}^{3}$, similar to that from the lattice QCD calculations [22].

Partons with transverse momenta $p_{T}>p_{0}(2 \mathrm{GeV})$ are mainly produced from initial hard collisions between nucleons. Their distributions can be determined from the pQCD calculation that includes the Cronin effect and radiative energy loss as described below.

In "elementary" hadron-hadron $(N+N)$ collisions, high $p_{T}$ minijet parton production can be evaluated using the perturbative QCD (pQCD) factorization approach [23]. This leads to a differential cross section that is expressed as a convolution of the measured parton distribution functions [24] $f_{\alpha / N}\left(x_{\alpha}, Q_{\alpha}^{2}\right)$ for the interacting partons $(\alpha=a, b)$ with the elementary parton-parton cross sections $d \sigma^{(a b \rightarrow c d)} / d \hat{t}$ 
[25,26]:

$$
\begin{aligned}
\frac{d \sigma_{\mathrm{jet}}}{d y d^{2} \boldsymbol{p}_{T}}= & K_{\mathrm{NLO}} \sum_{a b c d} \int d x_{a} d x_{b} \int d^{2} \boldsymbol{k}_{T a} d^{2} \boldsymbol{k}_{T b} \\
& \times f\left(\boldsymbol{k}_{T a}\right) f\left(\boldsymbol{k}_{T b}\right) f_{a / p}\left(x_{a}, Q_{a}^{2}\right) f_{b / p}\left(x_{b}, Q_{b}^{2}\right) \\
& \times \frac{\hat{s}}{\pi} \frac{d \sigma^{(a b \rightarrow c d)}}{d \hat{t}} \delta(\hat{s}+\hat{u}+\hat{t}) .
\end{aligned}
$$

In the above, $x_{a}$ and $x_{b}$ are the initial momentum fractions carried by the interacting partons. For nucleon-nucleon collisions, we use phenomenological smearing $\left\langle\boldsymbol{k}_{T}^{2}\right\rangle=1.8 \mathrm{GeV}^{2} / \mathrm{c}^{2}$ via a normalized Gaussian distribution function $f\left(\boldsymbol{k}_{T}\right)$ to mimic next-to-leading order and soft gluon resummation effects.

The $K_{\mathrm{NLO}}$ factor in Eq. (5) is determined from $p p$ collisions [27] by introducing the fragmentation function $D_{h / c}\left(z, Q_{c}^{2}\right)$ for minijet parton $c$ into hadrons of flavor $h$, i.e.,

$$
\frac{d \sigma_{\mathrm{had}}}{d y d^{2} \boldsymbol{p}_{T}}=\sum_{\mathrm{jet}} \int d z_{c} \frac{d \sigma_{\mathrm{jet}}\left(\boldsymbol{p}_{T} / z_{c}\right)}{d y d^{2} \boldsymbol{p}_{T}} \frac{D_{h / c}\left(z_{c}, Q_{c}^{2}\right)}{z_{c}^{2}},
$$

where $z_{c}=p_{h} / p_{c}$ is the momentum fraction carried by the observed hadron, and $Q_{c}=p_{h} / z_{c}$ is the momentum scale for the fragmentation process. Using the fragmentation functions of Ref. [28] for pions that are determined from $e^{+} e^{-}, e p$, and $p \bar{p}$ collisions, we find that the measured pion spectrum in $p p$ collisions at $\sqrt{s_{N N}}=62 \mathrm{GeV}$ [29] can be reproduced with a $p_{T}$-dependent $K_{\mathrm{NLO}}$ in Eq.(5). Similar $p_{T}$ dependence of the $K_{\mathrm{NLO}}$ factor for pion and direct photon production in $p p$ collisions has been previously found in Ref. [30]. Thus, in the calculation of the partonic spectra, we use $K_{\mathrm{NLO}}=2-0.33\left(6-p_{T}\right), 2<p_{T}<6 \mathrm{GeV}$, and $K_{\mathrm{NLO}}=2$ for $p_{T}>6 \mathrm{GeV}$.

In the presence of cold and hot nuclear matter, the multiple elastic, inelastic, and coherent scattering of the incoming and outgoing partons modifies the perturbative cross sections in Eq. (5). The calculated corrections can be systematically incorporated in the pQCD factorization approach [31]. For example, transverse momentum diffusion in $p+A$ collisions leads to the Cronin effect [27]. In $A+A$ reactions, the dominant nuclear effect is the energy loss of energetic jets propagating through the quark-gluon plasma, and the dominant energy loss mechanism is via medium-induced gluon bremsstrahlung. To first order in the mean number of scatterings, the radiative spectrum and the average energy loss, calculated in the Gyulassy-Levai-Vitev approach [32] for the case of Bjorken expansion [33], reads

$$
\begin{aligned}
& \omega \frac{d N_{\text {ind. }}^{g}}{d \omega} \approx C_{R} \frac{9 \pi \alpha_{s}^{3}}{4} \frac{1}{A_{\perp}} \frac{d N^{\text {parton }}}{d y} \\
& \times \begin{cases}\frac{L}{\omega}+\cdots, & \omega \gg \mu^{2} L / 2 \\
\frac{6}{\pi \mu^{2}} \ln \frac{\mu^{2} L}{2 \omega}+\cdots, & \omega \ll \mu^{2} L / 2\end{cases} \\
& \frac{\Delta E}{E} \approx \frac{1}{E} C_{R} \frac{9 \pi \alpha_{s}^{3}}{4} \frac{1}{A_{\perp}} \frac{d N^{\text {parton }}}{d y} L \\
& \times\left(\ln \frac{2 E}{\mu^{2} L}+\frac{3}{\pi}+\cdots\right),
\end{aligned}
$$

where $C_{R}=4 / 3$ (3) for quark (gluon) jets, $A_{\perp}$ is the transverse size of the interaction region, and $L$ is the distance traversed by the jets. We note that for the purpose of calculating the attenuation of the single inclusive hadronic spectra, $1+1 D$ expansion approximates well the $3+1 D$ result.

Eqs. (7) and (8) illustrate qualitatively the behavior of the energy loss as a function of the system size, density, and partonic energy. Quantitative jet quenching analysis requires numerical evaluation of the radiative spectra and the mean energy loss as in Refs. [26,27]. In the Poisson approximation of independent gluon emission, the probability $P(\epsilon)$ for fractional energy loss $\epsilon=\sum_{i} \omega_{i} / E$ due to multiple bremsstrahlung can be generated from the radiative spectrum as in Ref. [34]. Along the lines of Ref. [26], we choose a suppression factor that corresponds to an initial parton density $d N_{\text {parton }} / d y=$ 725. The resulting quenched minijet distributions are used for the parton distributions at $p_{T}>p_{0}$ in the coalescence process.

Since the probability for a minijet parton to coalesce with other partons to form a hadron is quite small, fragmentation remains the dominant mechanism for hadronization of minijets. It is thus a good approximation to neglect the correction due to competition from the coalescence [3,4] in evaluating the fragmentation contribution to hadron production from minijets. Besides their contribution to pions using the fragmentation functions of Ref. [28] that are determined from $e^{+} e^{-}, e p$, and $p \bar{p}$ collisions, we evaluate that to baryons such as $p$ and $\bar{p}$ based on a string-inspired parametrization of their fragmentation functions (see Refs. [35,36]). We anticipate that the latter better matches the nonzero baryon number $(B>0)$ for an average partonic scattering in the $A+A$ reaction than fragmentation functions based on the analysis of $e^{+} e^{-}$ annihilation with $B=0$. The resulting $p$ and $\bar{p}$ spectra are thus sensitive to the quark and antiquark distributions in the nucleon. This perturbatively computed hadron spectrum is referred to as the fragmentation component of the hadron spectrum.

We note that application of the energy loss calculations alone for neutral pions at $\sqrt{s}=62 \mathrm{GeV}$ in $\mathrm{Au}+\mathrm{Au}$ collisions at RHIC [26] yields a factor 2-3 suppression with only a moderate dependence on $p_{T}$. The medium-induced non-Abelian bremsstrahlung thus plays an important role in modifying the relative importance of the $\mathrm{pQCD}$ fragmentation mechanism when compared to the low $p_{T}$ non perturbative approaches such as string and baryon junction mechanisms $[36,37]$, relativistic hydrodynamics $[38,39]$, and quark coalescence $[3-5,18]$.

To obtain hadron transverse momentum spectra, we evaluate the multidimensional integral in Eq. (1) by the Monte Carlo method using test particles that are distributed uniformly in the fireball according to the distribution function and geometry described in this paper. Details of this method can be found in Ref. [4]. As in our studies for $\mathrm{Au}+\mathrm{Au}$ collision at center of mass energy $\sqrt{s_{N N}}=200 \mathrm{GeV}$, we include quark coalescence into stable hadrons such as $\pi, p$, and $\bar{p}$ as well as unstable resonances such as $\rho, \omega, \Delta$, and $K^{\star}$. Decays of the resonances are included, and they modify the spectra of stable hadrons mainly at $p_{T}<2 \mathrm{GeV}$, leading to a better agreement with experimental data at $\sqrt{s_{N N}}=200 \mathrm{GeV}$. 


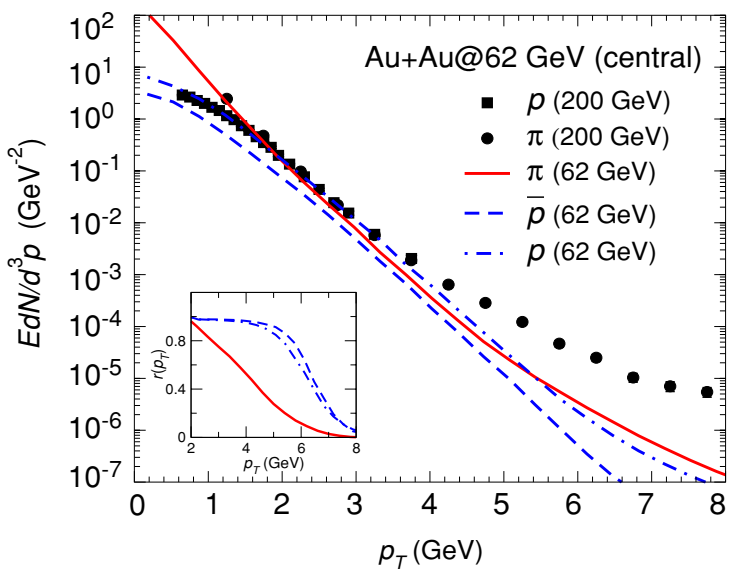

FIG. 1. (Color online) Hadron transverse momentum spectra from central $\mathrm{Au}+\mathrm{Au}$ collisions at $\sqrt{s_{N N}}=62 \mathrm{GeV}$. Predicted results are for pions (solid line), protons (dash-dotted line), and antiprotons (dashed line). Experimental $\pi^{0}$ (filled circles) and proton (open squares) data at $\sqrt{s_{N N}}=200 \mathrm{GeV}[40,42]$ are shown for reference. Inset: The ratio $r\left(p_{T}\right)$ of the contribution from quark coalescence to that from both quark coalescence and minijet fragmentation for $\pi$ (solid line), $p$ (dash-dotted line), and $\bar{p}$ (dashed line).

In Fig. 1, we show predicted $p_{T}$ spectra of pions (solid line), protons (dash-dotted line), and antiprotons (dashed line) including both coalescence and fragmentation contributions for $\mathrm{Au}+\mathrm{Au}$ collisions at $\sqrt{s_{N N}}=62 \mathrm{GeV}$. For comparison, we also show the experimental pion (filled circles) and proton (open squares) data at $\sqrt{s_{N N}}=200 \mathrm{GeV}$ from the PHENIX collaboration [40]. At low $p_{T}$, both pion and proton spectra are similar at the two energies. This is not surprising as the total charged particle multiplicity, which is dominated by low $p_{T}$ particles, is only reduced by about $20 \%$ as the collision energy decreases from 200 to $62 \mathrm{GeV}$. At higher $p_{T}$, the pion spectrum depends mostly on the fragmentation of quenched minijets. With respect to the case at $200 \mathrm{GeV}$, the quenching mechanism is somewhat less effective due to a lower value of the initial gluon density [26]. Preliminary data from the PHOBOS experiment on the suppression factor for charged particles have, indeed, confirmed such a trend [41]. On the other hand, less minijet partons are initially produced at $\sqrt{s_{N N}}=62 \mathrm{GeV}$ than at $\sqrt{s_{N N}}=200 \mathrm{GeV}$, and their transverse momentum distribution is also steeper, which amplifies the quenching effect at high $p_{T}$. The interplay between the nonperturbative and perturbative yields results in a $\pi$ spectrum that is similar to the one at $\sqrt{s_{N N}}=200 \mathrm{GeV}$ around $p_{T} \simeq 2.5 \mathrm{GeV}$ but falls down more quickly as shown in Fig. 1.

Figure 1 further shows that although pions dominate at low $p_{T}$, more protons are produced at intermediate $p_{T}$ up to $5.5 \mathrm{GeV}$. The antiproton yield also becomes comparable to the pion yield at intermediate transverse momenta. Since the minijet fragmentation gives the proton and antiproton spectra that are about a factor of 4 lower than the pion spectrum, the large proton and antiproton yields at intermediate transverse momenta are mainly due to contributions from the quark coalescence. This is further illustrated in the inset of Fig. 1, where the ratio $r\left(p_{T}\right)$ of the contribution from quark

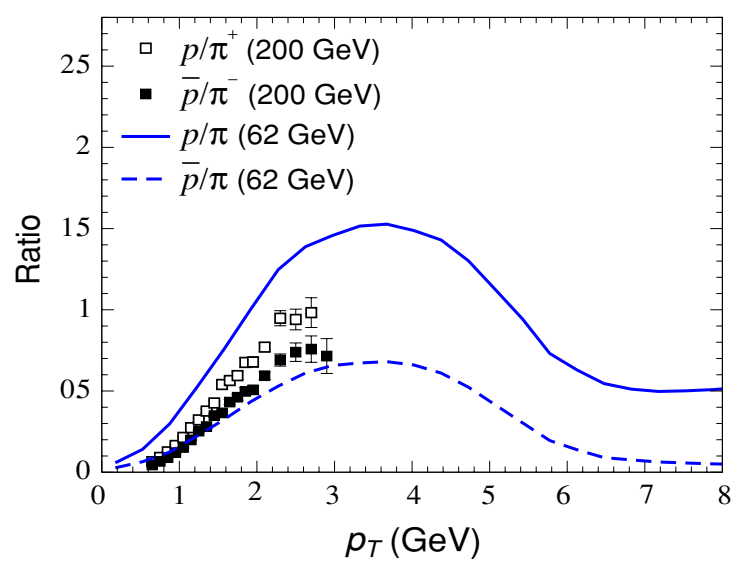

FIG. 2. (Color online) Same as Fig. 1 for the ratios of proton (solid line) and antiproton (dashed line) to pion spectra.

coalescence to that from both quark coalescence and minijet fragmentation is shown for $\pi$ (solid line), $p$ (dash-dotted line), and $\bar{p}$ (dashed line). It is seen that the quark coalescence contribution to proton production at intermediate transverse momenta overtakes the suppressed proton spectrum from minijet fragmentation up to $p_{T}<6.5 \mathrm{GeV}$, while for pions it is dominant only up to $p_{T}<4 \mathrm{GeV}$. We note that as in our previous study on $\mathrm{Au}+\mathrm{Au}$ collisions at $\sqrt{s_{N N}}=$ $200 \mathrm{GeV}[3,4]$, most intermediate transverse momentum hadrons are produced from the coalescence of minijet partons with thermal partons.

The predicted $p / \pi$ ratio in central $\mathrm{Au}+\mathrm{Au}$ collisions at $\sqrt{s_{N N}}=62 \mathrm{GeV}$ is shown in Fig. 2, and it reaches a value of about 1.5 at $p_{T} \sim 3.5 \mathrm{GeV}$. For comparison, the $p / \pi$ ratio of about 1 was measured in collisions at $\sqrt{s_{N N}}=200 \mathrm{GeV}$ as shown by open squares for the experimental data [42]. The large value at lower collision energy is due to the larger difference between the slopes of the spectra from fragmentation and coalescence in collisions at $\sqrt{s_{N N}}=62 \mathrm{GeV}$ than that at $\sqrt{s_{N N}}=200 \mathrm{GeV}$. Also shown in Fig. 2 is the $\bar{p} / \pi$ ratio at a collision energy of $\sqrt{s_{N N}}=62 \mathrm{GeV}$. It can reach a maximum value of about 0.7 , which is close to the value at $\sqrt{s_{N N}}=200 \mathrm{GeV}$, shown by filled squares for the experimental data, but at slightly larger $p_{T}$, as a result of the steeper $\pi$ spectrum from the fragmentation contribution. Because of the difference in the baryon chemical potentials, a larger difference between the $\bar{p} / \pi$ and $p / \pi$ ratios is seen at $\sqrt{s_{N N}}=62 \mathrm{GeV}$ than at $200 \mathrm{GeV}$.

Using a hadronization model based on quark coalescence and minijet fragmentation, we predicted the transverse momentum spectra of pions, protons, and antiprotons for central $\mathrm{Au}+\mathrm{Au}$ collisions at $\sqrt{s_{N N}}=62 \mathrm{GeV}$. We found a larger enhancement of the $p / \pi$ ratio with respect to the one seen in nuclear collisions at $\sqrt{s_{N N}}=200 \mathrm{GeV}$. Our result is mainly due to a steeper pion spectrum that is dominated by minijet fragmentation already at $p_{T} \simeq 4 \mathrm{GeV}$, and a proton spectrum that is instead still dominated by contributions from the quark coalescence up to $p_{T} \simeq 6.5 \mathrm{GeV}$. Experimental confirmation of this prediction will provide stronger evidence for quark coalescence as a likely nonperturbative mechanism for hadronization, especially for protons (and in general the 
baryons) at transverse momenta up to about $6 \mathrm{GeV}$. This in turn will also facilitate the determination of the characteristic energy at which the quark-gluon plasma is formed in heavy ion reactions.

Our results depend on the properties of the bulk partonic matter at the moment of hadronization and the amount of minijet quenching. In the present study, they are determined from extrapolations based on available data at $\sqrt{s_{N N}}=$ $17 \mathrm{GeV}$ from SPS as well as $\sqrt{s_{N N}}=130$ and $200 \mathrm{GeV}$ from RHIC. With upcoming experimental data on the total transverse energy, baryon chemical potential, and minijet suppression factor in collisions at $\sqrt{s_{N N}}=62 \mathrm{GeV}$, more reliable predictions on hadron spectra and their ratios can be obtained. Our work, however, represents an important first step in investigating the interplay between the nonperturbative and perturbative hadron production mechanisms at intermediate RHIC energies.

We are grateful to Zhangbu $\mathrm{Xu}$ for helpful comments on an earlier version of this paper. This paper was based on work supported by the U. S. National Science Foundation under Grant No. PHY-0098805, the Welch Foundation under Grant No. A-1358 (C. M. K. and V. G.) and the J. R. Oppenheimer grant of the Los Alamos National Laboratory (I. V.).
[1] S. A. Voloshin, Nucl. Phys. A715, 379 (2003).

[2] R. C. Hwa and C. B. Yang, Phys. Rev. C 67, 034902 (2003); Phys. Rev. Lett. 90, 212301 (2003).

[3] V. Greco, C. M. Ko, and P. Lévai, Phys. Rev. Lett. 90, 202302 (2003).

[4] V. Greco, C. M. Ko, and P. Lévai, Phys. Rev. C 68, 034904 (2003).

[5] R. J. Fries, B. Müller, C. Nonaka, and S. A. Bass, Phys. Rev. Lett. 90, 202303 (2003); Phys. Rev. C 68, 044902 (2003).

[6] D. Molnar and S. A. Voloshin, Phys. Rev. Lett. 91, 092301 (2003).

[7] Z. W. Lin and D. Molnar, Phys. Rev. C 68, 044901 (2003).

[8] V. Greco, C. M. Ko, and R. Rapp, Phys. Lett. B595, 202 (2004).

[9] V. Greco and C. M. Ko, Phys. Rev. C 70, 024901 (2004).

[10] K. Adcox et al., Phys. Rev. Lett. 88, 242301 (2002).

[11] S. S. Adler et al., Phys. Rev. Lett. 91, 182301 (2002).

[12] J. Adams et al., Phys. Rev. Lett. 92, 052302 (2003).

[13] T. S. Biró, P. Lévai, and J. Zimányi, Phys. Lett. B347, 6 (1995); J. Phys. G 28, 1561 (2002).

[14] P. Csizmadia and P. Lévai, Phys. Rev. C 61, 031903(R) (2000); J. Phys. G 28, 1997 (2002).

[15] C. Adler et al., Phys. Rev. Lett. 90, 082302 (2003); S. S. Adler, et al., nucl-ex/0408007.

[16] B. Zhang, C. M. Ko, B. A. Li, and Z. Lin, Phys. Rev. C 61, 067901 (2000); 62, 054905 (2000); 65, 054909 (2002); Z. Lin, S. Pal, C. M. Ko, B. A. Li, and B. Zhang, ibid. 64, 011902(R) (2001); Nucl. Phys. A498, 375c (2002); Z. Lin and C. M. Ko, Phys. Rev. C 65, 034904 (2002); Z. Lin, C. M. Ko, and S. Pal, Phys. Rev. Lett. 89, 152301 (2002); L. W. Chen, C. M. Ko, and Z. Lin, Phys. Rev. C 69, 031901(R) (2004); L. W. Chen, V. Greco, C. M. Ko, and P. Kolb, Phys. Lett. B605, 95 (2005); Z. Lin, C. M. Ko, B. A. Li, B. Zhang, and S. Pal, nucl-th/0411110.

[17] D. Molnar and M. Gyulassy, Nucl. Phys. A697, 495 (2002); Nucl. Phys. (erratum) A703, 893 (2002).
[18] D. Molnar, J. Phys. G 30, S1239 (2004).

[19] S. A. Bass, B. Muller, and D. K. Srivastava, J. Phys. G 30, S1283 (2004).

[20] Z. Xu and C. Greiner, hep-ph/0406278.

[21] S. S. Adler et al., Phys. Rev. C 71, 034908 (2005).

[22] F. Karsch and E. Laermann, hep-lat/0305025.

[23] J. C. Collins, D. E. Soper, and G. Sterman, Adv. Ser. Direct. High Energy Phys. 5, 1 (1988).

[24] M. Gluck, E. Reya, and A. Vogt, Eur. Phys. J. C 5, 461 (1998).

[25] J. F. Owens, Rev. Mod. Phys. 59, 465 (1987).

[26] I. Vitev, Phys. Lett. B606, 303 (2005).

[27] I. Vitev and M. Gyulassy, Phys. Rev. Lett. 89, 252301 (2002).

[28] J. Binnewies, B. A. Kniehl, and G. Kramer, Z. Phys. C 65, 471 (1995).

[29] D. d'Enterria, J. Phys. G 31, S491 (2005).

[30] G. G. Barnafoldi, G. I. Fai, P. Levai, G. Papp, and Y. Zhang, J. Phys. G 27, 1767 (2001).

[31] I. Vitev, J. Phys. G 30, S791 (2004); hep-ph/0409297.

[32] M. Gyulassy, P. Lévai, and I. Vitev, Phys. Rev. Lett. 85, 5535 (2000); Nucl. Phys. B594, 371 (2001); B571, 197 (2000).

[33] M. Gyulassy, I. Vitev, and X. N. Wang, Phys. Rev. Lett. 86, 2537 (2001).

[34] P. Lévai et al., Nucl. Phys. A698, 631 (2002); M. Gyulassy, P. Lévai, and I. Vitev, Phys. Lett. B538, 282 (2002).

[35] X. N. Wang, Phys. Rev. C 58, 2321 (1998).

[36] I. Vitev and M. Gyulassy, Phys. Rev. C 65, 041902(R) (2002).

[37] S. E. Vance and M. Gyulassy, Phys. Rev. Lett. 83, 1735 (1999).

[38] U. Heinz, AIP Conf. Proc. 739, 163 (2005); P. Kolb, nucl-th/ 0407066.

[39] E. Shuryak, Prog. Part. Nucl. Phys. 53, 273 (2004).

[40] S. S. Adler et al., Phys. Rev. Lett. 91, 072301 (2003).

[41] B. B. Back et al., Phys. Rev. Lett. 94, 082304 (2005).

[42] S. S. Adler et al., Phys. Rev. C 69, 034909 (2004). 